\title{
Victimización y perpetración de la violencia de pareja adolescente: Un modelo predictivo
}

\author{
Victimization and perpetration of the violence of \\ adolescent couple: A predictive model
}

\author{
Jaime Humberto MORENO-MÉNDEZ1 ${ }^{\text {ID }}$ 0000-0003-4087-6309 \\ María Margarita ROZO-SÁNCHEZ1 ${ }^{\text {ID } 0000-0003-3893-8216}$ \\ Sandra Jimena PERDOMO-ESCOBAR ${ }^{1}$ iD) 0000-0002-8171-8529 \\ Bertha Lucía AVENDAÑO-PRIETO2 ${ }^{\text {ID } 0000-0002-8136-5380}$
}

\section{Resumen}

La evidencia ha mostrado un incremento de la violencia en las relaciones de pareja, lo cual amerita identificar los factores que la predicen. El objetivo fue establecer un modelo predictivo de las características sociodemográficas, psicopatológicas y del consumo de alcohol sobre la victimización y la perpetración de la violencia de pareja adolescente. La muestra fue de 599 participantes, entre 13 y 19 años, de instituciones educativas públicas y privadas de Bogotá, Colombia. 327 eran del sexo femenino $(M=16.9 ; D E=1.45)$ y 272 del sexo masculino $(M=17.35 ; D E=1.35)$. Se aplicó el Cuestionario de Autoinforme de Variables Psicológicas, el Inventario de Síntomas, el Cuestionario de Identificación de los Trastornos debidos al Consumo de Alcohol, y el Inventario de Conflicto en las Relaciones en el Noviazgo Adolescente. El consumo de alcohol y los síntomas psicopatológicos predicen en el sexo femenino la victimización, y en el sexo masculino la perpetración de la violencia de pareja.

Palabras clave: Perpetración; Validez predictiva; Victimización; Violencia de pareja.

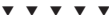

1 Universidad Católica de Colombia, Facultad de Psicología, Línea de Investigación Psicología Clínica de la Salud y de las Adicciones. Av. Caracas, 46-72, 110221, Bogotá D.C., Colombia. Correspondencia para/Correspondence to: J.H. MORENO-MÉNDEZ. E-mail: $<$ jhmoreno@ucatolica.edu.co>

2 Universidad Católica de Colombia, Facultad de Psicología, Línea de Investigación Métodos aplicados a las Ciencias del Comportamiento. Bogotá, D.C., Colombia.

Apoyo: Universidad Católica de Colombia (Code 411001)

Artículo es resultado de la investigación institucional "Características sociodemográficas y problemas de adaptación predictores de la victimización y perpetración de la violencia en parejas adolescentes” Universidad Católica de Colombia, 2018.

$\boldsymbol{\nabla} \mathbf{v}$

Como citar este artículo/How to cite this article

Moreno-Méndez, J. H., Rozo-Sánchez, M. M., Perdomo-Escobar, S. J., \& Avendaño-Prieto, B. L. (2019). Victimización y perpetración de la violencia de pareja adolescente: Un modelo predictivo. Estudos de Psicologia (Campinas), 36, el80146. http://dx.doi.org/10.1590/1982$0275201936 \mathrm{e} 180146$ 


\begin{abstract}
The evidence has shown an increase of violence in people's relationships that deserves identification of the underlying factors. The objective of this study was to establish a predictive model of sociodemographic, psychopathological and alcohol abuse characteristics about victimization and perpetration of violence in adolescent relationships. A sample composed of 599 participants between 13 and 19 years of age from public and private educational institutions in Bogotá, Colombia, was selected, in which 327 participants were female $(M=16.9 ; S D=1.45)$ and 272 male $(M=$ 17.35; SD = 1.35). The Self-report Questionnaire on Psychological Variables, Symptom Inventory, Alcohol Use Disorders Identification Questionnaire, and Conflict Inventory in Teen Dating were applied. Use of alcohol and psychopathological symptoms predict victimization in female adolescents and the perpetration of violence by male partners.
\end{abstract}

Keywords: Perpetration; Predictive validity; Victimization; Male partner's violence.

La violencia en parejas adolescentes es un fenómeno mundial que se ha constituido en un problema de salud pública por las consecuencias físicas, emocionales y sociales que ocasionan en las víctimas, tales como inflamación crónica, consumo de sustancias, problemas en salud sexual y reproductiva, depresión e ideación suicida (Decker et al., 2014; Karlsson, Calvert, Hernández-Rodríguez, Weston, \& Temple, 2018). La violencia es un problema de derechos humanos, por cuanto afecta el normal desarrollo de las personas involucradas en la dinámica de dicha violencia (Exner-Cortens 2014). Debido a lo anterior, es fundamental identificar los factores predictivos de la violencia en la pareja adolescente, tanto en las víctimas como en los perpetradores, con el fin de comprender dicha violencia y proponer estrategias para su prevención.

La violencia de pareja adolescente ha sido definida por Parker y Kim (2018) como la agresión de tipo físico, emocional, sexual, verbal y el comportamiento amenazante que ocurre en una relación de pareja entre adolescentes. También puede dirigirse hacia exparejas, ya sea cara a cara o de manera on-line (Priebe, Mitchell, \& Finkelhor, 2013; Rodríguez-Domínguez, Durán-Segura, \& Martínez-Pecino, 2018).

Decker et al. (2014) hallaron en países como Nigeria, India, Sudáfrica, Estados Unidos y China, una heterogeneidad significativa en la prevalencia de violencia en pareja entre mujeres adolescentes, con y sin compañero estable, con vulnerabilidad socioeconómica, que varió entre el $25.0 \%$ de las mujeres que alguna vez tuvieron pareja frente al $10.0 \%$ de las que no la tenían. Smith et al. (2018) reportaron una prevalencia del $35.8 \%$ en victimización y 33.0\% en perpetración. Cuevas, Sabina, y Bell (2014) encontraron una prevalencia del $14.8 \%$ de violencia psicológica de pareja. En Colombia, a octubre de 2018 se reportaron 3.009 casos de violencia de pareja en el rango de los 15 a los 19 años (Instituto Nacional de Medicina Legal y Ciencias Forenses, 2018).

La evidencia empírica resalta que entre las manifestaciones más frecuentes de violencia de pareja están la verbal-emocional seguida de la violencia sexual en ambos miembros de la pareja como una forma de afrontar los conflictos. También las chicas tienden a manifestar más agresión verbal-emocional y física que los chicos, lo cual parece estar asociado a que los chicos tienden a minimizar y tener un menor reconocimiento de la agresividad que expresan y a que las chicas expresan más libremente que cometen dicho tipo de violencia, dada la mayor tolerancia social al respecto (Pazos, Oliva, \& Hernando, 2014; Rodríguez-Domínguez, et al., 2018).

Diferentes perspectivas dan cuenta del fenómeno de la violencia de pareja adolescente. La teoría del aprendizaje social de Bandura (1977) es consistente con la hipótesis de transmisión intergeneracional, mediante la cual se establece que un estilo interpersonal coercitivo y aversivo es aprendido de experiencias previas de violencia en la familia de origen.

Adicionalmente, se ha planteado una asociación entre la violencia de pareja en adolescentes y sus resultados adversos, mediada por el estilo romántico de apego (Exner-Cortens, 2014; Miga, Hare, Allen, \& 2 Manning, 2010). Wekerle y Wolfe (1999) reportan que los adolescentes con sentimientos de inseguridad en 
las relaciones afectivas significativas tienden a experimentar celos, labilidad emocional y síntomas obsesivos como una forma de expresar un estilo de apego ansioso ambivalente. Se considera que una historia de maltrato infantil tiende a generar modelos de apego inseguros, de acuerdo a dimensiones basadas en relaciones de dominación del hombre y subordinación de la mujer y de perpetración-victimización de la violencia en las relaciones románticas.

Varios estudios han reportado que la agresividad, los bajos niveles de regulación emocional, la ira, la ansiedad en las niñas y el alto consumo de alcohol en los niños, previos a la violencia de pareja, eran fuertes predictores de la misma (Fernández-González, Calvete, Orue, \& Echezarraga, 2018; Foshee et al., 2015; Jennings et al., 2017).

Por otro lado, Miga et al. (2010) determinaron que la ansiedad en adolescentes a los 14 años predice un comportamiento verbalmente agresivo hacia las parejas románticas a los 18 años, bajo la forma de inducción de la culpa y manipulación. Por su parte Liu, Cohen, Schulz, y Waldinger (2011) puntualizaron que la tendencia a la perpetración de la violencia en los hombres está mediada por el apego temeroso y la somatización.

Igualmente, Temple et al. (2016) reportan que los adolescentes con síntomas de ansiedad y depresión pueden tener mayor riesgo de evidenciar violencia hacia su pareja, dadas las dificultades con la regulación emocional asociadas a dichas problemáticas.

Otros estudios han determinado que el historial de problemas de salud mental y presencia de agresiones físicas en la familia, el establecimiento de relaciones con pares con problemas de comportamiento y la intimidación física directa hacia compañeros en la adolescencia temprana, eran factores de predisposición de violencia de pareja (Foshee et al., 2015; Park \& Kim, 2018; Reidy, Early, \& Holland, 2017; Ruiz-Pérez, Rodríguez-Barranco, Cervilla, \& Ricci-Cabello, 2018; Tenkorang \& Owusu, 2018).

En otras investigaciones se ha determinado que en parejas de adolescentes que estaban bajo los efectos de sustancias psicoactivas resultado del consumo indebido de alcohol estas eran predictores de violencia en la pareja (Epstein-Ngo et al., 2014). Asimismo, las creencias y actitudes fundamentadas en el sexismo hostil, los celos románticos y las discusiones sobre sexo a través de medios virtuales, se encuentran vinculadas a la perpetración de la violencia de adolescentes hacia sus parejas románticas a edades tempranas (Rodríguez-Domínguez et al., 2018).

Mackay, Bowen, Walker, y O’Doherty (2018) y Vitoria-Estruch, Romero-Martínez, Lila-Murillo, y Moya-Albiol (2018) concluyeron que los hombres que evidencian un alto consumo de alcohol y que maltratan a sus parejas tienen un mayor deterioro en procesos atencionales, en memoria de trabajo, flexibilidad cognitiva, planeación, toma de decisiones, toma de perspectiva, así como mayores fallas en habilidades para decodificar las emociones.

Los procesos de inhibición también se ven comprometidos en hombres que presentan síntomas psicóticos, dado que las dificultades de autocontrol, la impulsividad e inflexibilidad cognitiva, así como el deterioro de la empatía emocional, propician la agresión hacia sus parejas (Darmedru, Demily, \& Franck, 2017; Ntounas et al., 2018; Oakley, Harris, Fahy, Murphy, \& Picchioni, 2016).

Por otro lado, diferentes estudios reportan que las víctimas de violencia de pareja adolescente tienen menor autoconcepto (Penado \& Rodicio-García 2017; Smith et al., 2018). Esta vulnerabilidad es más evidente en adolescentes entre 16 y 17 años, como lo muestra el estudio de Penado y Rodicio-García, (2017), el cual estableció que la violencia sexual interactúa significativamente con el autoconcepto emocional, social y físico en ambos géneros.

También el estrés es otra variable predictora de victimización de violencia de pareja adolescente (Smith et al., 2018), mientras que otros estudios han indicado que presenciar la violencia entre los padres era el 
principal factor de vulnerabilidad de victimización (Madruga, Viana, Rigacci-Abdalla, Caetano, \& Laranjeira, 2017; Parker \& Kim, 2018; Reidy et al., 2017; Tenkorang \& Owusu, 2018).

Miga et al. (2010) adicionalmente reportan que los adolescentes ansiosos y con apegos inseguros tenían un mayor riesgo de ser víctimas de agresión verbal por parte de sus parejas románticas cuatro años después de iniciada la relación. Esto se puede explicar porque cuando la adolescente se retira física y emocionalmente para evitar discusiones en la relación, dicha actitud puede ser percibida de manera frustrante y evocar respuestas emocionales y de comportamiento violentas en su pareja. Liu et al. (2011) señalan que cuando las mujeres neutralizan los sentimientos de enojo como una manera compensatoria de evitar el conflicto con su pareja, pueden experimentar sensaciones corporales que terminan por conducir a quejas somáticas adicionales.

En cuanto a los factores protectores, Parker y Kim (2018) comprobaron que en los vecindarios donde hay relaciones de apoyo, estas facilitan la prevención de la violencia en parejas adolescentes. FernándezGonzález et al. (2018) señalaron que la capacidad para regular las propias emociones es un factor protector de la violencia de pareja en los adolescentes.

Con base en lo anterior, el objetivo del estudio consistió en establecer un modelo predictivo de las características sociodemográficas, psicopatológicas y del consumo de alcohol sobre la victimización y la perpetración de la violencia en las relaciones de pareja en adolescentes.

\section{Método}

\section{Participantes}

Adolescentes entre 13 y 19 años, vinculados a instituciones educativas públicas y privadas de la ciudad de Bogotá, Colombia. Se seleccionó una muestra bajo un procedimiento no probabilístico, con un nivel de confianza del 95\% y un margen de error del 5\%, mediante el programa estadístico GPower. Los criterios de inclusión fueron: (a) haber tenido o tener una relación amorosa de al menos un mes de duración; (b) tener entre 13 y 19 años; (c) ser soltero o soltera; y (d) contar con la autorización de la institución educativa para realizar el estudio, con el consentimiento escrito de los padres y con el asentimiento del adolescente.

La muestra final estuvo conformada por 599 adolescentes, 327 (54.5\%) del sexo femenino $(M=$ 16.9; $D E=1.45)$ y $272(45.5 \%)$ del sexo masculino $(M=17.35 ; D E=1.35)$. En relación con los adolescentes, el $54.8 \%$ cursaba el último grado de bachillerato, el $40.4 \%$ pertenecía al estrato socioeconómico bajo, el $93.8 \%$ se identificó como heterosexual, el $36.1 \%$ reportó haber tenido una pareja y el $69.6 \%$ tenía diez o más meses de duración con la relación actual. En cuanto a las adolescentes, el 38.2\% cursaba el último grado de bachillerato, el $43.4 \%$ pertenecía al estrato socioeconómico bajo, el $92.0 \%$ se identificó como heterosexual, el 39.0\% reportó haber tenido una pareja y el 64.5\% tenía 10 o más meses de duración con la relación actual (Tabla 1).

\section{Instrumentos}

Conflict in Adolescent Dating Relationships Inventory (CADRI), versión española (Fernández-Fuertes et al., 2006). Diseñado originalmente por Wolfe et al. (2001) evalúa cinco factores sobre perpetración y victimización de la violencia física, verbal-emocional, relacional, sexual y amenazas en la pareja en los últimos 12 meses, por medio de 35 díadas de ítems tipo Likert con cuatro opciones de respuesta: "nunca" (0), "rara vez" (1), "a veces" (2) y "con frecuencia" (3). Los índices de consistencia interna oscilan entre 0.51 y 0.79 ,

4 con un valor alfa de 0.86 para todo el cuestionario. El análisis factorial exploratorio evidenció una estructura 
Tabla 1

Características sociodemográficas de los participantes

\begin{tabular}{|c|c|c|}
\hline Variables & $\%$ Masculino $(n=272)$ & $\%$ Femenino $(n=327)$ \\
\hline \multicolumn{3}{|l|}{ Edad } \\
\hline 13 & 1.8 & 1.5 \\
\hline 14 & 3.1 & 2.8 \\
\hline 15 & 11.9 & 10.5 \\
\hline 16 & 23.5 & 24.1 \\
\hline 17 & 19.3 & 22.7 \\
\hline 18 & 26.3 & 28.2 \\
\hline 19 & 14.1 & 15.6 \\
\hline \multicolumn{3}{|l|}{ Escolaridad } \\
\hline Sexto & 0.6 & 0.6 \\
\hline Séptimo & 0.9 & 0.9 \\
\hline Octavo & 1.8 & 1.8 \\
\hline Noveno & 18.7 & 18.7 \\
\hline Décimo & 23.2 & 23.2 \\
\hline Once & 54.8 & 38.2 \\
\hline \multicolumn{3}{|c|}{ Estrato socioeconómico } \\
\hline Bajo (1 y 2 ) & 40.4 & 43.4 \\
\hline Medio (3 y 4) & 34.9 & 36.5 \\
\hline Alto $\quad(5$ y 6$)$ & 15.4 & 20.1 \\
\hline \multicolumn{3}{|c|}{ Orientación sexual } \\
\hline Heterosexual & 93.8 & 92 \\
\hline Homosexual & 6 & 7.5 \\
\hline Bisexual & 0.2 & 0.5 \\
\hline \multicolumn{3}{|c|}{ Número de parejas } \\
\hline Una & 36.1 & 39 \\
\hline Dos & 20 & 36 \\
\hline Tres & 22.9 & 18 \\
\hline Cuatro & 8 & 5 \\
\hline Cinco o más & 13 & 2 \\
\hline \multicolumn{3}{|c|}{ Número de meses con la relación actual } \\
\hline Uno & 5.9 & 5.9 \\
\hline Dos & 5.1 & 4.9 \\
\hline Tres & 4 & 6 \\
\hline Cuatro & 3.1 & 3.7 \\
\hline Cinco & 3.7 & 3.3 \\
\hline Seis & 3.4 & 3.8 \\
\hline Siete & 1.2 & 2.5 \\
\hline Ocho & 2.1 & 2.5 \\
\hline Nueve & 1.8 & 2.9 \\
\hline Diez o más & 69.6 & 64.5 \\
\hline
\end{tabular}


de seis factores que explicaban el $54.23 \%$ de la varianza, aunque un análisis forzado mostró que cinco factores podrían explicar al menos el 50.00\% de la varianza (Fernández-Fuertes et al., 2006).

Lista de Síntomas SCL-90-R (Derogatis, 1988), versión española (De las Cuevas et al., 1991). Es un instrumento que permite evaluar la presencia de 90 síntomas psicopatológicos en las últimas dos semanas, agrupados en las subescalas de somatización, obsesiones y compulsiones, sensibilidad interpersonal, depresión, ansiedad, hostilidad, ansiedad fóbica, ideación paranoide y psicoticismo. La forma de calificación es tipo Likert con cinco alternativas de respuesta: "nada en absoluto"; (0), "un poco" (1); "moderadamente" (2); "bastante" (3) y "mucho o extremadamente" (4). Los autores de la versión española establecieron una estructura de ocho factores que consideraron homologables a los de la versión original y que explicaban el $32,46 \%$ de la varianza en 570 españoles de ambos sexos entre 18 y 74 años.

Cuestionario de auto informe de variables psicológicas (Rey-Anacona, 2012). Se diseñó con el propósito de obtener información de los adolescentes sobre algunas características sociodemográficas tales como edad, sexo, escolaridad (grado 8 a 11 de bachillerato) estrato (bajo, medio y alto), orientación sexual, número de parejas y tiempo con la relación actual. Tenía 49 ítems con diferentes opciones de respuesta. El instrumento fue revisado a nivel metodológico y de contenido por expertos y probado con una muestra de adolescentes varones y mujeres.

Cuestionario de Identificación de los Trastornos debidos al Consumo de Alcohol (Alcohol Use Disorders Identification Test AUDIT), Organización Mundial de la Salud (1993). El instrumento tiene un método simple de screening del consumo excesivo de alcohol para uso internacional. Está compuesto por 10 preguntas acerca del consumo de alcohol, las cuales se puntúan en una escala de 0 a 4 . La calificación está agrupada en cuatro categorías: $\sin$ consumo $=0$, Consumo moderado $=1$ a 7; Consumo de riesgo $=8$ a 15; Consumo perjudicial $=16$ a 19 y Consumo dependiente $=20$ a 40. En cuanto a los datos de consistencia y fiabilidad, el instrumento tiene un Alfa de Cronbach $=0.82$ para la escala total y correlaciones positivas entre todos los reactivos y superiores a 0.3 en los reactivos de cada subescala (Riveros, Vera, Gantiva, \& Torres, 2018). El instrumento se aplicó de forma auto aplicada.

\section{Procedimiento}

La investigación se llevó a cabo en las siguientes fases: en la primera fase se obtuvo la autorización del estudio por parte del Comité de Ética de la Universidad Católica de Colombia, según acta de inicio del proyecto del 22 de enero de 2018. Para la selección de los participantes y la administración de instrumentos, se solicitó la autorización para la realización del estudio en diferentes instituciones educativas. En la segunda fase se convocó a los padres y madres para explicarles junto con sus hijos el proyecto, para así obtener su consentimiento y asentimiento firmados. Posteriormente, se procedió a aplicarles a los adolescentes los instrumentos de manera grupal. Finalmente, en la tercera fase, se analizaron los resultados. En reconocimiento por la participación en el estudio, a los adolescentes se les hizo entrega de unas cartillas psicoeducativas para la promoción de relaciones armónicas con sus parejas y a las instituciones se les hizo entrega de los resultados generales del estudio.

\section{Análisis de datos}

El análisis de datos tuvo el propósito de analizar la relación entre las características sociodemográficas, psicopatológicas y el consumo de alcohol, como variables predictoras, sobre la victimización y la perpetración de la violencia en las relaciones de pareja en adolescentes, como variables de criterio. Se utilizó un modelo de ecuaciones estructurales, previo cumplimiento de los supuestos requeridos para el uso de esta técnica. 
Para el análisis de bondad de ajuste del modelo se siguieron las indicaciones señaladas en la literatura (Pilatti et al., 2012), se utilizaron los estadísticos chi-cuadrado (CMIN), la razón de chi-cuadrado sobre los grados de libertad (CMIN/DF), el índice de ajuste comparativo (CFI), el índice de bondad del ajuste global (GFI), el índice no normalizado de ajuste o Tucker Lewis (TLI), el Incremental Fit Index (IFI) y el error cuadrado de aproximación (RMSEA).

El estadístico chi-cuadrado indica el ajuste absoluto del modelo, pero es muy sensible al tamaño de la muestra; por consiguiente, se utilizó la razón de chi cuadrado sobre los grados de libertad. Valores inferiores a 3 indican un buen ajuste. Los índices CFI y GFI varían entre 0 y 1; 0 indica ausencia de ajuste y 1 ajuste óptimo. Valores de 0.95 o superiores son considerados excelentes y valores superiores a 0.90 sugieren un ajuste aceptable del modelo a los datos. El índice RMSEA es considerado óptimo cuando sus valores son de 0.05 o inferiores y aceptables en el rango 0.05 - 0.08. El índice no normalizado de ajuste o Tucker Lewis (TLI), es considerado aceptable con puntuaciones mayores o iguales a 0.90 (Escobedo, Hernández, Estebane, \& Martínez, 2016), y el IFI, con valores superiores a 0.90 es indicativo de buen ajuste (Leal-Costa, TiradoGonzález, Rodríguez-Marín; \& Vander-Hofstadt-Román, 2016).

\section{Resultados}

En cuanto al nivel descriptivo de cada una de las dimensiones de los cuatro instrumentos aplicados a los adolescentes de ambos sexos, se encontró que los síntomas psicopatológicos que más prevalecieron en los participantes del sexo masculino fueron la somatización, con un 19.5\%, seguidos de los síntomas fóbicos, con un $11.0 \%$. En los adolescentes del sexo femenino fueron el psicoticismo, con un $17.1 \%$, seguidos de la somatización, con un $14.1 \%$.

En relación con la variable consumo de alcohol, se encontró una mayor proporción de consumo moderado, tanto en adolescentes del sexo femenino, con un 60.6\%, como en adolescentes del sexo masculino, con un $59.9 \%$. Llama la atención que el $16.2 \%$ de los adolescentes y el $11.0 \%$ de las adolescentes tiene un consumo de riesgo. De acuerdo con el autoinforme de variables psicológicas, se encontró que la edad de inicio del consumo de alcohol en las adolescentes fue entre 11 y 12 años en el $52.0 \%$ de los casos y en el $50.0 \%$ en los adolescentes. En los últimos 30 días, el $46.0 \%$ de los adolescentes y el $42.2 \%$ de las adolescentes reportaron haber consumido alcohol entre 1 y 2 veces. El 18.4\% de los adolescentes y el 15.3\% de las adolescentes reportó haberse embriagado entre 1 y 2 veces durante dicho periodo.

Los resultados reportados sobre la violencia de pareja dan cuenta de más altos porcentajes en la perpetración de la violencia verbal por parte de las mujeres hacia su pareja con un $90.8 \%$ y de los hombres hacia su pareja con un $79.0 \%$. Así mismo, se encontraron altos porcentajes en la victimización de la violencia verbal en las mujeres con un $89.9 \%$ y en los hombres con un $80.9 \%$. Por otro lado, se determinó un porcentaje considerable de perpetración de violencia sexual en los hombres, con un $44.9 \%$, mientras que en las mujeres se estableció un $\mathbf{4 5 . 9 \%}$ en victimización de la violencia sexual.

Para el análisis de la relación entre las variables, se hizo uso de las ecuaciones estructurales. Los índices de bondad de ajuste obtenidos con el software AMOS 23 fueron los siguientes: la razón entre el valor de chi-cuadrado $\left(\chi^{2}\right)$ y el número de grados de libertad correspondientes fueron: femenino $\left(\chi^{2} / \mathrm{gl}=1.89\right)$; masculino ( $\left.\chi^{2} / \mathrm{gl}=1.53\right)$. El índice global de ajuste (Goodness of Fit Index), femenino $(\mathrm{GFI}=0.91)$; masculino $(\mathrm{GFI}=0.91)$ y el índice de ajuste comparativo (Comparative fit index), femenino $(\mathrm{CFI}=0.94)$; masculino $(\mathrm{CFI}=0.96)$. El CFI compara la mejora en el ajuste del modelo en cuestión a un modelo nulo en el que todos los ítems son independientes y no se permiten factores comunes; este índice es de los más utilizados, puesto que es poco sensible al tamaño de la muestra (Sternberg, Prieto, \& Castejón, 2000). El RMSEA = 0.05 fue encontrado en el sexo femenino, mientras que en el sexo masculino fue RMSEA $=0.04$, error cuadrado de 
aproximación a las raíces medias (Root Mean Square Error of Approximation). El intervalo de confianza del RMSEA, osciló entre 0.04 y 0.06 en el sexo femenino y para el sexo masculino fue 0.03; 0.05.

La Figura 1 muestra el diagrama de la estructura con los respectivos indicadores de las relaciones entre las variables y sus dimensiones en los adolescentes del sexo femenino. Las cargas factoriales del consumo de alcohol oscilan entre 0.37 y 0.75 , las cargas factoriales de los síntomas psicopatológicos oscilan entre 0.66 y 0.91 y las cargas factoriales de la violencia de pareja adolescente oscilan entre 0.56 y 0.77 .

Ninguna de las condiciones sociodemográficas aportó significativamente al modelo. Las variables más importantes que explican la violencia de pareja adolescente fueron el consumo de alcohol, medido a través del AUDIT, específicamente la dimensión 3 que indica la frecuencia en que se consumen seis o más bebidas alcohólicas en una misma ocasión con un valor estimado de 0.75 . Entre el consumo de alcohol y la violencia de pareja adolescente, medida con el CADRI, se encontró un efecto positivo de la primera variable sobre la segunda, dado que la relación entre estas dos variables tiene una carga de valor estimado de 0.28 , con un nivel de significancia de 0.00. En otras palabras, el peso de regresión para el consumo de alcohol en la predicción de la victimización de la violencia de pareja adolescente es significativamente diferente de cero en el nivel de 0.05 (dos colas).

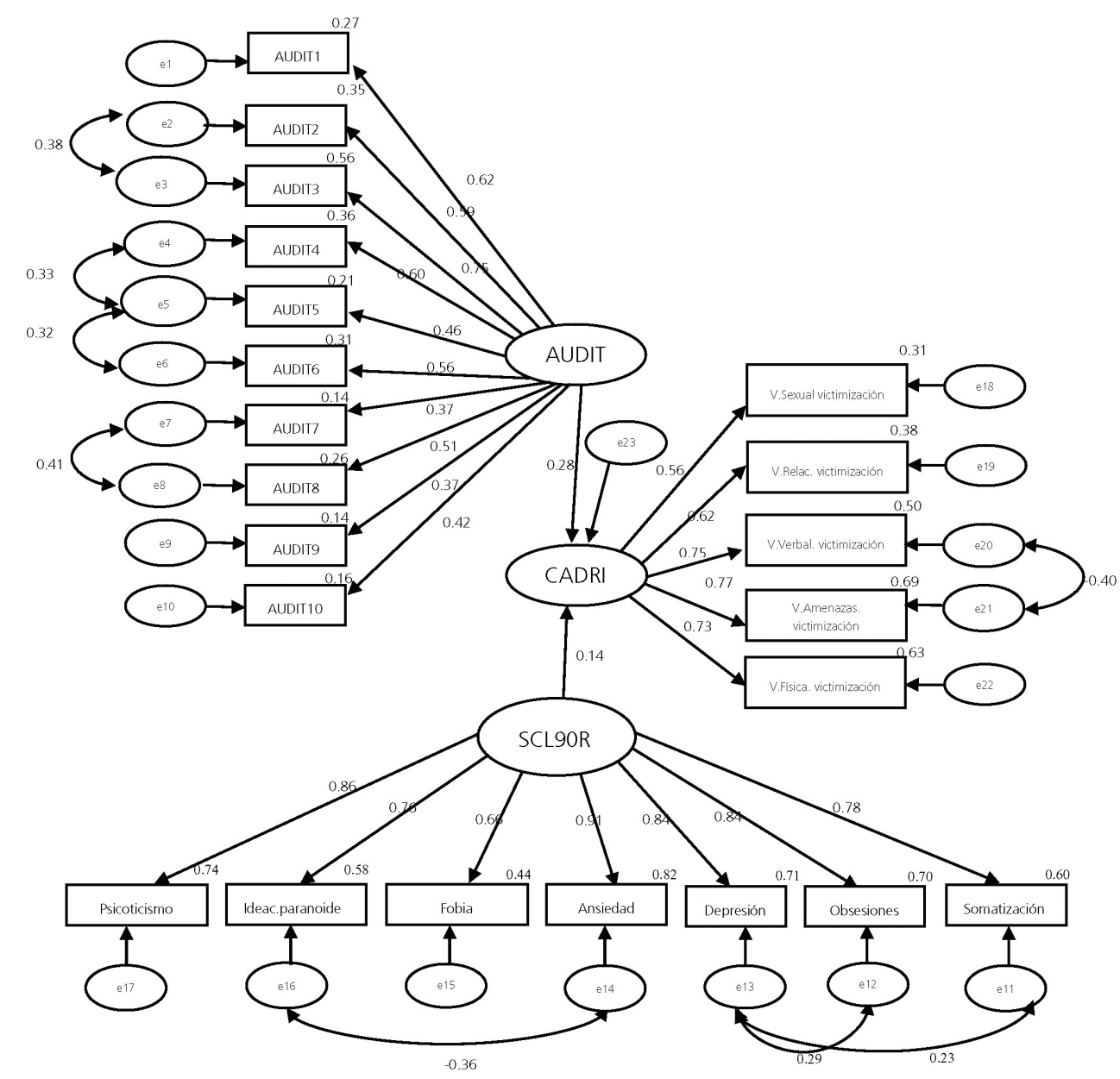

Figura 1. Modelo predictivo en el sexo femenino del consumo de alcohol y los síntomas psicopatológicos sobre la violencia de pareja adolescente. Adaptado del Programa Estadístico para las Ciencias Sociales (SPSS) versión 22 (IMB, 2013). CARDI: Conflict in Adolescent Dating Relationships Inventory. 
Sobre la relación entre los síntomas psicopatológicos y la violencia de pareja adolescente, el efecto de la primera variable sobre la segunda tiene una carga de valor estimado de 0.14 , con un nivel de significancia de 0.02. Los indicadores de bondad de ajuste presentados en la Tabla 1 evidencian el grado en el que los datos se ajustan al modelo propuesto, confirmando su validez. Los síntomas psicopatológicos, medidos con el SCL 90R, de psicoticismo, ansiedad, obsesiones y compulsiones, depresión, ideación paranoide y somatización, con valores estimados entre 0.76 y 0.91 , predicen la victimización de la violencia de pareja física, verbal, emocional, sexual y amenazas en las adolescentes.

La Figura 2 muestra el modelo predictivo de las variables consumo de alcohol y síntomas psicopatológicos sobre la perpetración de la violencia de pareja en los adolescentes del sexo masculino. La carga factorial del consumo de alcohol fes 0.28 . Las cargas factoriales de los síntomas psicopatológicos oscilan entre 0.67 y 0.87 y las cargas factoriales de la violencia de pareja adolescente oscilan entre 0.51 y 0.71 .

Ninguna de las condiciones sociodemográficas aportó significativamente al modelo. Entre el consumo de alcohol y la violencia de pareja adolescente medida con el CADRI se encontró un efecto positivo de la primera variable sobre la segunda, dado que la relación entre estas dos variables tiene una carga de valor estimado de 0.28 , con un nivel de significancia de 0.00 . En otras palabras, el peso de regresión para el consumo de alcohol en la predicción de la perpetración de la violencia de pareja adolescente es significativamente diferente de cero en el nivel de 0.05 (dos colas).

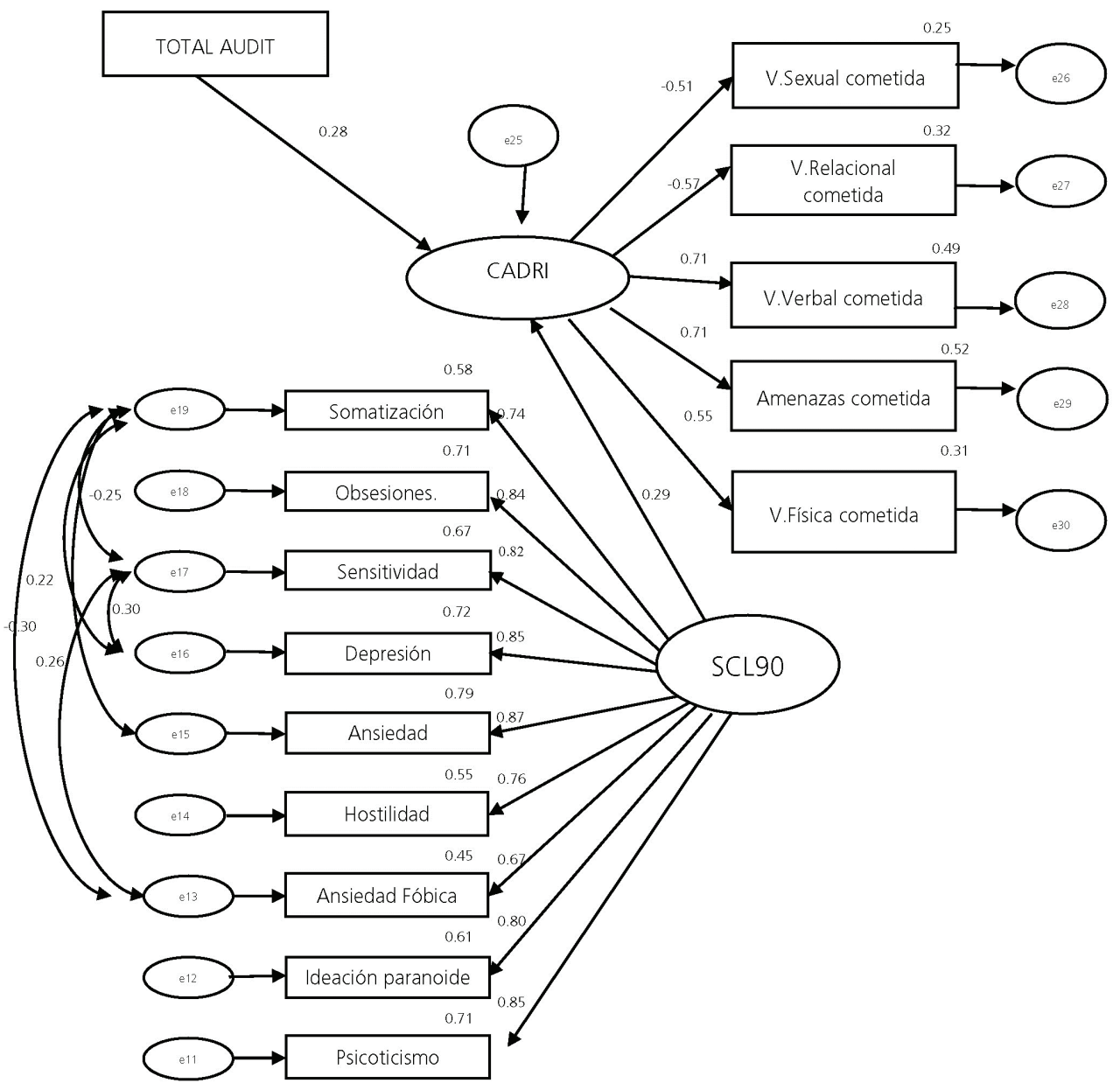

Figura 2. Modelo predictivo en el sexo masculino del consumo de alcohol y los síntomas psicopatológicos sobre la violencia de pareja adolescente. Adaptado del Programa Estadístico para las Ciencias Sociales (SPSS) versión 22 (IMB, 2013). 
En cuanto a la relación entre los síntomas psicopatológicos y la violencia de pareja adolescente, el efecto de la primera variable sobre la segunda tiene una carga de valor estimado de 0.29 , con un nivel de significancia de 0.02. Los indicadores de bondad de ajuste presentados en la tabla evidencian el grado en el que los datos se ajustan al modelo propuesto, confirmando su validez. Los síntomas psicopatológicos de somatización, ansiedad, sensibilidad interpersonal, depresión, ansiedad fóbica y psicoticismo, medidos con el SCL 90R con valores estimados entre 0.67 y 0.87 , predicen la perpetración de la violencia de pareja física, verbal, emocional, sexual y amenazas en los adolescentes.

\section{Discusión}

El objetivo del presente estudio consistió en establecer un modelo predictivo entre las condiciones sociodemográficas, el consumo de alcohol y los síntomas psicopatológicos sobre la perpetración y la victimización de la violencia de pareja adolescente. Al respecto, se estableció que el consumo de seis o más bebidas alcohólicas en una misma ocasión es la dimensión que más explica el consumo de alcohol, mientras que la ansiedad es la que más explica los síntomas psicopatológicos.

En el modelo se encontró que el consumo de alcohol predice la victimización de la violencia de pareja en las adolescentes. Resultado que puede ser explicado porque los adolescentes tienden a incrementar el consumo de alcohol en relaciones románticas conflictivas, lo que a su vez puede exacerbar sentimientos de ira y llevar a una escalada de la tensión en la relación y culminar con la victimización de la mujer (Baker, 2016; Espelage, Davis, Basile, Rostad, \& Leemis, 2018). Llama la atención que el promedio de edad de inicio del consumo es a los 12 años, lo cual denota un comienzo a temprana edad y que en la actualidad hay un consumo de nivel de moderado a de riesgo. Esto se ha determinado en estudios previos en los que se evidencia que el consumo problemático se constituye en un factor de riesgo para la violencia de pareja en los adolescentes (Fernández-González et al., 2018; Foshee et al., 2015; Jennings et al., 2017).

Los síntomas psicopatológicos predicen la victimización de la violencia de pareja en las adolescentes. Acorde con Parker y Kim (2018) y Rodríguez-Domínguez et al. (2018), los hallazgos del presente estudio confirman que las adolescentes evaluadas experimentan violencia de pareja de tipo emocional, relacional, sexual, verbal y física. De igual forma, los datos coinciden con Smith et al. (2018), en el sentido de que en el sexo femenino hay mayor victimización que perpetración de dicho tipo de violencia. Acorde con Cuevas et al. (2014), se concluye que en las adolescentes hay una superposición de diferentes formas de victimización de violencia de pareja.

La ansiedad en las adolescentes desempeña un papel predictivo significativo en la victimización de la violencia en las relaciones de pareja, lo cual puede ser explicado en consideración a que los adolescentes que establecen relaciones románticas con una pareja con un estilo de apego ansioso tienden a su vez a experimentar victimización verbal y física. También las adolescentes idealizan la violencia con base en el amor romántico y les restan importancia a los comportamientos violentos de sus parejas (Soldevila, Domínguez, Giordano, Fuentes, \& Consolini, 2012), lo que denota la tendencia a legitimar roles de dominación en el hombre y de sumisión en la mujer. Esto es consistente con Miga et al. (2010) y Wekerle y Wolfe (1999), quienes explican que la adolescente se retira física y emocionalmente con el propósito de minimizar el daño personal porque tiende a asumir la culpa de la agresión en la relación y para evitar discusiones con su pareja. Igualmente, evidencia temor a defender su derecho a no ser vulnerada, lo cual genera una percepción de indefensión y sentimientos ansiosos y depresivos. Dicha actitud puede ser percibida de manera frustrante y evocar respuestas emocionales y de comportamiento violentas en su pareja. Otra posible explicación es que las mujeres adolescentes tienen dificultades para reconocer que son víctimas de malos tratos (Pazos et al., 2014; Vizcarra, Poo, \& Donoso, 2013). 
Por otro lado, los datos del estudio no reportaron una predicción de las condiciones sociodemográficas sobre la violencia de pareja en adolescentes, como el haber observado episodios de violencia doméstica en su niñez. Estos resultados son diferentes a los previamente encontrados, en los que se ha resaltado que presenciar la violencia entre los padres es el principal factor de vulnerabilidad y de victimización (Madruga et al., 2017; Parker \& Kim, 2018; Reidy, et al., 2017; Tenkorang \& Owusu, 2018).

Acorde con lo señalado por Rodríguez-Domínguez et al. (2018), los adolescentes del sexo masculino evidencian perpetración de violencia física, sexual, relacional, verbal y amenazas sobre sus parejas. Esto puede ser explicado según Wekerle y Wolfe (1999) en el sentido de que los adolescentes inseguros de sus relaciones afectivas significativas tienden a experimentar celos, labilidad emocional y síntomas obsesivos, lo cual los lleva a perpetrar violencia hacia sus parejas. A su vez, puede ser el resultado de una forma de expresión producto del aprendizaje social de las relaciones románticas de dominación del hombre y subordinación de la mujer. Los hombres son socializados para comportarse de manera agresiva, dominante y con poca expresión emocional, mientras que a las mujeres se les enseña a asumir un rol pasivo, complaciente y dependiente de sus parejas (Wekerle \& Wolfe, 1999).

Se estableció un papel predictivo de las variables consumo de alcohol y síntomas psicopatológicos sobre la perpetración de la violencia de pareja en adolescentes del sexo masculino. Este es consistente con lo determinado por otros estudios que reportan que el alto consumo de alcohol puede tener un impacto en los perpetradores en procesos atencionales, memoria de trabajo, flexibilidad cognitiva, planeamiento, toma de decisiones, toma de perspectiva, así como mayores fallas en habilidades para decodificar las emociones, lo que dificulta afrontar adecuadamente los conflictos en la relación de pareja (Fernández-González et al., 2018; Foshee et al., 2015; Jennings et al., 2017; Vitoria-Estruch et al., 2018).

En los resultados del presente estudio se estableció que la ansiedad y la depresión en los adolescentes predicen en los hombres la práctica de violencia hacia sus parejas románticas. Esto se puede explicar porque dichos problemas internalizantes tienden a ir acompañados de ira, irritabilidad y dificultades en la regulación emocional que terminan expresándose de forma violenta hacia la pareja para asegurarse que permanezca involucrada en la relación. (Judd, Schettler, Coryell, Akiskal, \& Fiedorowicz, 2013; Miga et al., 2010; Temple, 2016; Yu, Pepler, Van de Bongardt, Josephson, \& Connolly, 2018). Adicionalmente, los hombres evitan revelar la perpetración como una manera de negar el problema y su responsabilidad, así como para mantener el control sobre su pareja (Wekerle \& Wolfe, 1999; Yu et al., 2018).

También se encontró que los síntomas psicóticos y la ideación paranoide desempeñan en los hombres un papel predictor de la violencia de pareja, dadas las fallas en el procesamiento cognitivo y en el control de los impulsos (Darmedru et al., 2017; Ntounas et al., 2018; Oakley et al., 2016).

Desde la perspectiva clínica, para la prevención de la violencia de pareja en mujeres adolescentes se propone, por un lado, trabajar en la regulación de la conducta de consumo de alcohol y en el manejo de la ansiedad, de tal forma que les permita tener mayores estrategias de afrontamiento que contribuyan a prevenir la aparición de dicho fenómeno en las relaciones de pareja. Por otro lado, con los hombres se requiere incrementar su regulación emocional, mejorar la flexibilidad cognitiva y reforzar estrategias de autocontrol del consumo de alcohol.

Como limitaciones del presente estudio, podemos citar que solo se evaluó a la población de Bogotá, por lo cual es difícil generalizar los resultados a otras poblaciones; así mismo, la muestra no fue homogénea. Se sugiere para próximos estudios seguir investigando el papel que cumplen las variables sociodemográficas y familiares en el desarrollo y mantenimiento de la violencia de pareja adolescente.

\section{Contribuyentes}

Todos los autores contribuyeron al diseño y análisis de los datos del estudio. 


\section{Referencias}

Baker, C. K. (2016). Dating violence and substance use: Exploring the context of adolescent relationships. Journal of Interpersonal Violence, 31(5), 900-919. http://dx.doi.org/10.1177/0886260514556768

Bandura, A. (1977). Social learning theory. Englewood Cliffs: Prentice-Hall.

Cuevas, C. A., Sabina, C., \& Bell, K. A. (2014). Dating violence and interpersonal victimization among a national sample of latino youth, Journal of Adolescent Health, 55, 564-570. http://dx.doi.org/10.1016/j.jadohealth.2014.04.007

Darmedru, C., Demily, C., \& Franck, N. (2017). Cognitive remediation and social cognitive training for violence in schizophrenia: A systematic review. Psychiatry Research, 251, 266-274. http://dx.doi.org/10.1016/j. psychres.2016.12.062

Decker, M. R., Peitzmeier, S., Olumide, A., Acharya, R., Ojengbede, O., Covarrubias, L., ... Brahmbhatt, H. (2014). Prevalence and health impact of intimate partner violence and non-partner sexual violence among female adolescents aged 15 e 19 years in vulnerable urban environments: A multi-country study. Journal of Adolescent Health, 55, 58-67. http://dx.doi.org/10.1016/j.jadohealth.2014.08.022

De las Cuevas, C., González de Rivera, J. L., Henry-Benítez, M., Monterrey, A. L., Rodríguez-Pulido, F., \& Gracia Marco, R. (1991). Análisis factorial de la versión española del SCL-90-R en la población general. Anales de Psiquiatría, 7(3), 93-96. Recuperado el febrero 15, 2019 de http://www.psicoter.es/pdf/91_A098_20.pdf

Derogatis, L. R. (1988). Cuestionario de 90 sintomas SCL-90-R. Madrid: TEA.

Epstein-Ngo, Q. M., Walton, M. A., Chermack, S. T., Blow, F. C., Zimmerman, M. A., \& Cunningham, R. M. (2014). Eventlevel analysis of antecedents for youth violence: Comparison of dating violence with non-dating violence. Addictive Behaviors, 39, 350-353. http://dx.doi.org/10.1016/j.addbeh.2013.10.015

Escobedo, M. T., Hernández, J. A., Estebane, V., \& Martínez, G. (2016). Modelos de ecuaciones estructurales: Características, fases, construcción, aplicación y resultados. Ciencia \& Trabajo, 18(55), 16-22. http://dx.doi.org/10.4067/S071824492016000100004

Espelage, D. L., Davis, J. P., Basile, K. C., Rostad, W. L., \& Leemis, R. W. (2018). Alcohol, prescription drug misuse, sexual violence, and dating violence among high school youth. Journal of Adolescent Health, 63, 601-607. https://dx.doi. org/10.1016/j.jadohealth.2018.05.024

Exner-Cortens, D. (2014). Theory and teen dating violence victimization: Considering adolescent development. Developmental Review, 34, 168-188. Recuperado de http://dx.doi.org/10.1016/j.dr.2014.03.001

Fernández-Fuertes, A. A., Fuertes, A., \& Pulido, R. F. (2006). Evaluación de la violencia en las relaciones de pareja de los adolescentes. Validación del Conflict in Adolescent Dating Relationships Inventory (CADRI) - versión española. International Journal of Clinical and Health Psychology, 6(2), 339-358. Recuperado el febrero 14, 2019, de http:// www.redalyc.org/html/337/33760208/

Fernández-González, L., Calvete, E., Orue, I., \& Echezarraga, A. (2018). The role of emotional intelligence in the maintenance of adolescent dating violence perpetration. Personality and Individual Differences, 127, 68-73. https:// dx.doi.org/10.1016/j.paid.2018.01.038

Foshee, V. A., McNaughton, L., Tharp, A. T., Chang, L., Ennett, S. T., Simon, T., ... Suchindran, C. (2015). Shared longitudinal predictors of physical peer and dating violence. Journal of Adolescent Health, 56, 106-112 http://dx.doi. org/10.1016/j.jadohealth.2014.08.003

Instituto Nacional de Medicina Legal y Ciencias Forenses. (2018). Boletín estadístico mensual. Octubre 2018. Colombia: Subdirección de Servicios Forenses. Centro de Referencia Nacional sobre Violencia. Recuperado el febrero 8, 2019, de http://www.medicinalegal.gov.co/documents/20143/217010/Boletin+Estadistico+mes+de+Octubre+2018. pdf/5efb7c5b-f242-553f-4e54-7ed01d4da274

Jennings, W. G., Okeem, C., Piquero, A. R., Sellers, C. S., Theobald, D., Farrington, D. P. (2017). Dating and intimate partner violence among young persons ages 15-30: Evidence from a systematic review. Aggression and Violent Behavior, 33, 107-125. http://dx.doi.org/10.1016/j.avb.2017.01.007

Judd, L. L., Schettler, P. J., Coryell, W., Akiskal, H. S., \& Fiedorowicz, J. G. (2013). Overt irritability/anger in unipolar major depressive episodes: Past and current characteristics and implications for long-term course. Jama Psychiatry, 70(11), 1171-1180. http://dx.doi.org/10.1001/jamapsychiatry.2013.1957

Karlsson, M. E., Calvert, M., Hernandez-Rodriguez, J., Weston, R., \& Temple, J. R. (2018). Changes in acceptance of dating violence and physical dating violence victimization in a longitudinal study with teens. Child Abuse \& Neglect, 86, 123-135. http://dx.doi.org/10.1016/j.chiabu.2018.09.010 
Leal-Costa, C., Tirado-González, S., Rodríguez-Marín, J., \& Vander-Hofstadt-Román, C. J. (2016). Psychometric properties of the Health Professionals Communication Skills Scale (HP-CSS). International Journal of Clinical and Health Psychology, 16(1), 76-86. Retrieved February 15, 2019, from http://www.redalyc.org/articulo.oa?id=33743098008

Liu, L., Cohen, S., Schulz, M. S., \& Waldinger, R. J. (2011). Sources of somatization: Exploring the roles of insecurity in relationships and styles of anger experience and expression. Social Science \& Medicine, 73, 1436-1443. http://dx.doi. org/10.1016/j.socscimed.2011.07.034

Mackay, J., Bowen, E., Walker, K., \& O'Doherty L. (2018). Risk factors for female perpetrators of intimate partner violence within criminal justice settings: A systematic review. Aggression and Violent Behavior, 41, 128-146. http://dx.doi. org/10.1016/j.avb.2018.06.004

Madruga, C. S., Viana, M. C., Rigacci-Abdalla, R., Caetano, R., \& Laranjeira, R. (2017). Pathways from witnessing parental violence during childhood to involvement in intimate partner violence in adult life: The roles of depression and substance use. Drug and Alcohol Review, 36(1), 107-114. http://dx.doi.org/10.1111/dar.12514

Miga, E. M., Hare, A., Allen, J. P., \& Manning, N. (2010). The relation of insecure attachment states of mind and romantic attachment styles to adolescent aggression in romantic relationships. Attachment and Human Development, 12(5), 463-481. http://dx.doi.org/10.1080/14616734.2010.501971

Ntounas, P., Katsouli, A., Efstathiou, V., Pappas, D., Chatzimanolis, P., Touloumis, C., ... Douzenis, A. (2018). Comparative study of aggression - dangerousness on patients with paranoid schizophrenia: Focus on demographic data, PANSS, drug use and aggressiveness. International Journal of Law and Psychiatry, 60, 1-11. http://dx.doi.org/10.1016/j. ijlp.2018.06.001

Oakley, C., Harris, H., Fahy, T., Murphy, D., \& Picchioni, M. (2016). Childhood adversity and conduct disorder: A developmental pathway to violence in schizophrenia. Schizophrenia Research, 172, 54-59. Recuperado de http:// dx.doi.org/10.1016/j.schres.2016.01.047

Organización Mundial de la Salud. (1993). Cuestionario de Identificación de los Trastornos debidos al Consumo de Alcohol (AUDIT). Pautas para su utilización en atención primaria. Genebra: Autor.

Parker, S., \& Kim, S. (2018). The power of family and community factors in predicting dating violence: A meta-analysis. Aggression and Violent Behavior, 40, 19-28. http://dx.doi.org/10.1016/j.avb.2018.03.002

Pazos G. M., Oliva D. A., \& Hernando G. A. (2014). Violencia en relaciones de pareja de jóvenes y adolescentes. Revista Latinoamericana de Psicología, 46(3), 148-159. Recuperado el febrero 15, 2019, de http://www.scielo.org.co/scielo. php?script=sci_arttext\&pid=S0120-05342014000300002\&lng=en\&tlng= .

Penado, M., \& Rodicio-García, M. L. (2017). Análisis del autoconcepto en las víctimas de violencia de género entre adolescentes. Suma Psicológica, 24, 107-114. http://dx.doi.org/10.1016/j.sumpsi.2017.08.001

Pilatti, A., Godoy, J. C., \& Brussino, S. A. (2012). Adaptación de instrumentos entre culturas: Ejemplos de procedimientos seguidos para medir las expectativas hacia el alcohol en el ámbito argentino. Trastornos Adictivos, 14(2), 58-64. http://dx.doi.org/10.1016/S1575-0973(12)70045-4

Priebe, G., Mitchell, K., \& Finkelhor, D. (2013). To tell or not to tell? Youth's responses to unwanted Internet experiences. Cyberpsychology: Journal of Psychosocial Research on Cyberspace, 7(1). http://dx.doi.org/10.5817/CP2013-1-6

Reidy, D. E., Early, M. S., \& Holland, K. M. (2017). Boys are victims too? Sexual dating violence and injury among high-risk youth. Preventive Medicine, 101, 28-33. http://dx.doi.org/10.1016/j.ypmed.2017.05.018

Rey-Anacona, C. A. (2012). Estudio descriptivo comparativo de adolescentes varones y adolescentes mujeres que presentan trastorno disocial de inicio infantil y trastorno disocial de inicio adolescente. (Informe de investigación inédito) Tunja: Universidad Pedagógica y Tecnológica de Colombia.

Riveros, F., Vera, L. A., Gantiva, C., \& Torres, L. (2018). Adaptación del Cuestionario para Identificación de Trastornos Debidos al Consumo de Alcohol (AUDIT) en universitarios colombianos. Revista de Psicopatología y Psicología Clínica, 23, 231-238. http://dx.doi.org/10.5944/rppc.vol.23.num.3.2018.22258

Rodríguez-Domínguez, C., Durán-Segura, M., \& Martínez-Pecino, R. (2018). Ciberagresores en el noviazgo adolescente y su relación con la violencia psicológica, el sexismo y los celos. Health and Addictions, 18(1), 17-27. Htt://dx.doi. org/10.21134/haaj.v18i1.329

Ruiz-Pérez, I., Rodríguez-Barranco, M., Cervilla, J. A., \& Ricci-Cabello, I. (2018). Intimate partner violence and mental disorders: Co-occurrence and gender differences in a large cross-sectional population based study in Spain. Journal of Affective Disorders, 229, 69-78. http://dx.doi.org/10.1016/j.jad.2017.12.032

Smith, K., Cénata, J. M., Lapierrea, A., Dionb, J., Héberta, M., \& Côté, K. (2018). Cyber dating violence: Prevalence and correlates among high school students from small urban areas in Quebec. Journal of Affective Disorders, 234, 220-223. http://dx.doi.org/10.1016/j.jad.2018.02.043 
Soldevila, A., Domínguez, A., Giordano, R., Fuentes, S., \& Consolini, L. (2012). ¿Celos, amor, culpa o patología? Cómo perciben la violencia de género en sus relaciones de pareja los/as estudiantes de Trabajo Social. Actas del II Congreso Interdisciplinario sobre Género y Sociedad: 'Lo personal es político', 1(1). Córdoba: Universidad Nacional de Córdoba. Recuperado el enero 25, 2019 de http://www.ffyh.unc.edu.ar/piemg/noticias/ii-congreso-2012-actas.html

Sternberg, R. J., Prieto, M. D., \& Castejón, J. L. (2000). Análisis factorial confirmatorio del Sternberg Triarchic Abilities Test (nivel H) en una muestra española: Resultados preliminaries. Psicothema, 12, 642-647. Recuperado el febrero 15, 2019 de http://www.psicothema.com/pdf/384.pdf

Temple, J. R., Choi, H. J., Elmquist, J., Hecht, M., Miller-Day, M., Stuart, G. L., ... Wolford-Clevenger, C. (2016). Psychological abuse, mental health, and acceptance of dating violence among adolescents, Journal of Adolescent Health, 59, 197202. http://dx.doi.org/10.1016/j.jadohealth.2016.03.034

Tenkorang, E. Y., \& Owusu, A. Y. (2018). A life course understanding of domestic and intimate partner violence in Ghana. Child Abuse \& Neglect, 79, 384-394. https://dx.doi.org/10.1016/j.chiabu.2018.02.027

Vitoria-Estruch, S., Romero-Martínez, A., Lila-Murillo, M., \& Moya-Albiol, L. (2018). Differential cognitive profiles of intimate partner violence perpetrators based on alcohol consumption, Alcohol, 70, 61-71. http://dx.doi.org/10.1016/j. alcohol.2018.01.006

Vizcarra, M. B., Poo, A. M., \& Donoso, T. (2013). Programa educativo para la prevención de la violencia en el noviazgo. Revista de Psicología, 22(1), 48-61. http://dx.doi.org/10.5354/0719-0581.2013.27719

Wekerle, C., \& Wolfe, D. A. (1999). Dating violence in mid-adolescence: Theory, significance, and emerging prevention initiatives. Clinical Psychology Review, 19(4), 435-456. http://dx.doi.org/10.1016/S0272-7358(98)00091-9

Wolfe, D. A., Scott, K., Reitzel-Jaffe, D., Wekerle, C., Grasley, C., \& Pittman, A. L. (2001). Development and validation of the conflict in adolescent dating relationships inventory. Psychological Assessment, 13(2), 277-293. http://dx.doi. org/10.1037/1040-3590.13.2.277

Yu, R, Peplerb, D. J., Van de Bongardt, D., Josephsond, W. I., \& Connolly J. (2018). Internalizing symptoms and dating violence perpetration in adolescence. Journal of Adolescence, 69, 88-91. http://dx.doi.org/10.1016/j. adolescence.2018.09.008

Recibido: Febrero 5, 2019

Versión final: Septiembre 24, 2019

Aprobado: Octubre 1, 2019 